\title{
OPTIMIZING FUNCTIONAL DISTRIBUTION IN COMPLEX SYSTEM DESIGN
}

\author{
O. P. Dias ${ }^{1)}$, I. M. Teixeira ${ }^{2)}$, J. P. Teixeira ${ }^{2)}$, \\ L. B. Becker ${ }^{3)}$,C. E. Pereira ${ }^{4)}$
}

1) Escola Superior de Tecnologia, IPS, INESC, Portugal

2) Instituto Superior Técnico, UTL, INESC, Portugal

3) Institute of Computer Science, UFRGS, Porto Alegre, Brazil

4) Electrical Engineering Department, UFRGS, Porto Alegre, Brazil

\begin{abstract}
The purpose of this paper is to show that functionality distribution among embedded objects of a system architecture critically influences the quality of the resulting architecture. It is shown that gains can be achieved when functionality distribution is guided by optimization criteria, using an automatically generated centralized processing architecture. This architecture is modified in order to carry out distributed processing. Two alternatives are then used: first, functionality associated with data processing is removed from the processing class, to different external classes, manually. Second, the entire architecture is re-generated to comply with distributed processing. Object Oriented Paradigm and UML, in conjunction with an extension of data flow diagram (E-DFD) conveying timing information are used for system requirement formalization. SIMOO$R T$ is used as graphical interface for direct Use-Case and E-DFD diagram constructions and for simulation. SysObj is used for architecture generation and quality assessment. Resident Quality Metrics and Criteria are used. Metric values obtained for the different solutions prove that it is rewarding to carry out functionality distribution optimization.
\end{abstract}

\section{Introduction}

Electronic hardware/software (hw/sw) systems design is an increasingly difficult task due to high complex functionality and stringent quality requirements [1]. Additional difficulties result from the heterogeneous nature of most common solutions, based on the use of complex hw/sw modules and extensive reuse of pre-defined Intellectual Property (IP) cores $[2,3]$ provided by different suppliers, eventually using different specification languages. Moreover, functional and non-functional requirements (such

The original version of this chapter was revised: The copyright line was incorrect. This has been corrected. The Erratum to this chapter is available at DOI: 10.1007/978-0-387-35409-5_23 
as testability or dependability) must be taken into consideration [4,5]. In fact, implicit requirements that are not specifically related with desired functionality, influence the final implementation [5] and thus must be considered.

In general, several solutions, reflecting different design tradeoffs and corresponding to different architectures, satisfy user's requirements. A tradeoff implies that characteristics are compared and some are valued against others. However, not all designers equally value the same characteristics. Therefore, consensus on the 'best-fitted' architecture is difficult to reach. Quality Metrics (QMs) need to be defined, and decision criteria to support architecture selection have to be devised.

In fact, an adequate choice of system architecture is a key factor of success in product development. The authors have recently proposed a methodology for system architecture generation, selection and reconfiguration, together with a set of designoriented and test-oriented QMs and decision criteria [5-8]. The proposed methodology leads to architectural solutions for which cohesive, autonomous, loosely coupled and balanced objects (from the complexity and/or performance points of view) result. The characteristic of having objects with similar execution times can enhance system performance and increase the possibilities of parallel operation.

Another key issue in system design is the decision on either to concentrate or distribute intelligence, and in what measure [9]. In fact, not only economic reasons force the reuse of low-cost, existing components, but also embedded or external functionality leads to architectures more or less complex, with good or bad performance, and hard or easy to test. If an experienced design team develops an architecture and wants to evaluate the trade-off of moving part of the functionality to the system boundaries (i.e., to the dialoguing actors), how to evaluate it? How does the quality of the architecture is deteriorated (or not) by it? These are the questions addressed in this paper, which is organized as follows. In the section 2 , the architectural design approach is described. In section 3, the methodology supporting tools are presented and their main characteristics highlighted. In section 4, a case study is described and the architecture alternatives (for distributed versus concentrated intelligence), obtained according to two different strategies, are presented and compared. Finally, section 5 draws the main conclusions.

\section{Design Methodology}

As referred, a key issue in the design process is the choice of an adequate architecture. The architecture should lead to a low-cost solution, with high performance of the entire functionality, while satisfying quality and other nonfunctional requirements.

In most cases, experienced designers applying previously working solutions define system architecture. For sw design using the Object-Oriented $(\mathrm{OO})$ paradigm [10-12], the design team makes manually the empiric identification of all system classes and objects, according to a given textual description. At system level, the architecture should not incorporate a large number of top-level objects, to manage complexity [13]. The functionality (object methods, in OO semantics) assigned to 
each object should be such that balanced object complexity (and execution time) results, along with loose object associations; this latter feature corresponds to known sw quality metrics, such as object cohesion, coupling and autonomy [14,15]. Although the valued characteristics of a "good" architecture are those above mentioned, there is a limited effort to quantify the architecture quality. Unless obvious drawbacks in the proposed architecture become evident, the architecture is accepted, and the design flow continues. Similar considerations can be made in hw design, for which a strong reuse of pre-verified hw modules, or IP cores, emphasizes the trend of a priori object (module) definition [3]. The monolithic integration of embedded cores in SOCs (Systems on a Chip) severely restricts object accessibility, enhancing the need for an efficient use of DFT (Design for Testability) techniques. However, these usually emerge late in the design flow, when object behavior is mapped into a structural hw netlist of logic elements.

The authors recently proposed a methodology that supports system designers on the automatic architecture generation and formal quality assessment of derived architectures [5-9]. The underlying principle is to provide an automatic procedure to assign functionality to identifiable objects, leading to objects with the characteristics outlined above. In Fig. 1, the different phases of the methodology are depicted.

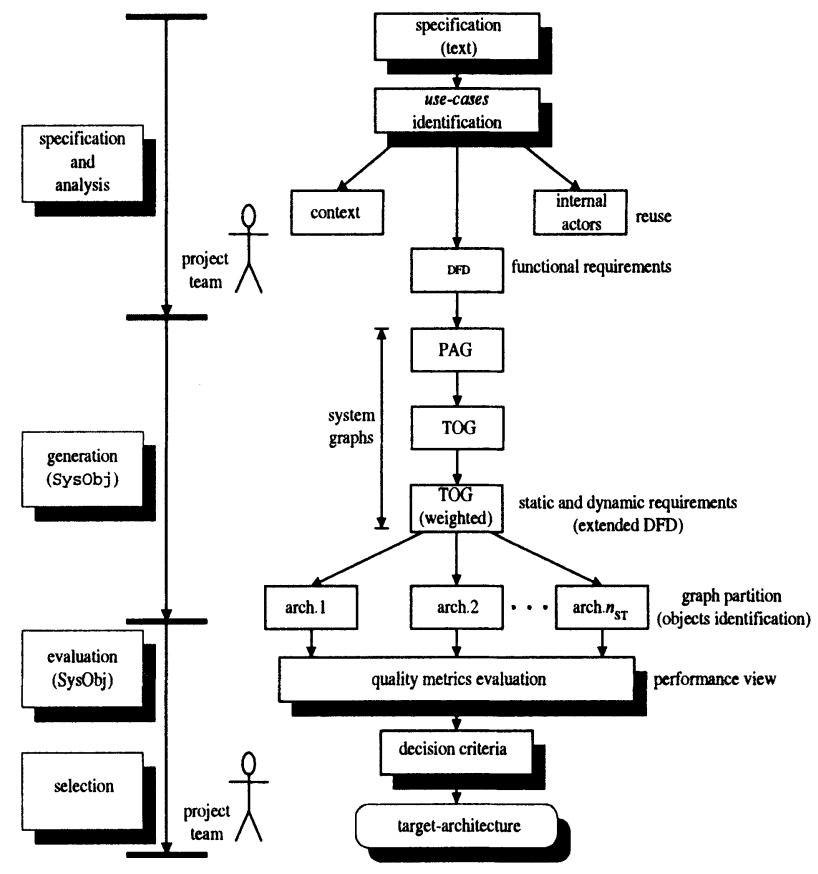

Fig. 1 - Steps of the proposed methodology

At specification level, Object-Oriented (OO) modeling techniques [10-12], together with the Unified Modeling Language [16] and an extension of Data Flow Diagrams (E-DFD) are used. Following UML, actors are identified (together with 
their attributes), as well as their interaction with the system under development. Usecase diagrams are used to model actors and system interaction, as well as the system's main functionality. Design economics usually makes mandatory the reuse of pre-defined macros, or cores (considered as internal, non-reconfigurable objects). If functional implementation is to be carried out in hw, such IP cores can be legacy cores, or provided by different IC vendors [2]. These are modeled as internal actors.

Nevertheless, there is always functionality that cannot be assigned to a semantically meaningful class (object). In this case, several classes can be generated to encompass this functionality. In our methodology, we propose that class definition be carried out based on formal constraints, provided by formally defined QMs and clustering criteria. By doing so, class generation becomes almost deterministic and corresponds to an optimum distribution of functionality according to a given set of parameters.

In order to obtain this optimum functional distribution a graph representation of functionality is used. In this TOG (Task-Oriented Graph), nodes are tasks and edges are associations between tasks. The graph is the formal mapping of an extended DFD, for which (1) processes represent the unassigned functionality and (2) data or control represent the corresponding attributes, conditioned by timing constraints (if this is required). We assume atomic processes, and define a task as the set of an atomic process and the single attribute it updates. Graph partitioning, according to different strategies and algorithms, will lead to different possible task clustering, enabling an optimal functionality distribution among classes. Each cluster of tasks is defined as an object. Design and test oriented metrics and decision criteria are used, a posteriori, to select the most adequate architecture. At present, graph partitioning strategies value object balancing (in terms of complexity, or performance) and mincut, in order to minimize object association and dependence.

The most adequate architecture is the one that better meets some desirable properties. This is evaluated by the values obtained for pre-defined quality metrics and criteria.The possibility of automatic architecture generation and quality assessment (by means of QM computation), guided by optimization criteria, open ways not only to assess the quality of an architectural solution proposed by a design team, but also to compare it with automatically generated solutions.

However, an additional usefulness of the proposed methodology emerges: the optimization of functional distribution (or assignment) to objects, when tradeoff analysis must be carried out, e.g., between distributed or centralized processing. In fact, in many industrial applications, e.g., in real-time automation systems, practical solutions can favor either distributed or centralized processing. In the case study presented in section 4 , the question is: should we use either smart (intelligent) sensors, and a network of less complex control systems, associated with each machine in a wine bottling production line, or local (dumb) sensors, and a network or more complex control systems?

In such case, if a 'good' architecture solution for centralized processing has been reached, the usual procedure is to manually move part of the processing from the system to the external actors. Conversely, if a 'good' solution has been derived for distributed processing, one can move part of the functionality into the system boundaries. However, are we moving from one optimized solution to another one? 
The claim we make in our work is that it ain't necessarily so, and often a better architectural solution can be derived. The proof is presented in section 4 .

\section{Tool Support}

The tool environment that supports the methodology integrates SIMOO-RT modeling tool [17,18] and SysObj [6]. A brief description of the main features of each individual tool is presented in next subsections.

\subsection{SIMOO-RT Modeling Tool}

SIMOO [17] is a framework for development of discrete object-oriented simulation models. Among the objectives proposed for this environment, there is the possibility to specify simulation models using rules that allows its use in the implementation of the control system for real entities. The representation of simulation entities by means of autonomous elements, based in the idea of active objects, suggests not only the definition of a distributed application but the construction of reusable entities library.

The SIMOO tool offers to the users a modeling tool called MET (Model Editing Tool), presented in fig. 2. The modeling approach adopted is hierarchical, allowing the definition of several levels of detail, since the first class of the model needs to be refined into lower-level classes, defining an aggregation relationship. Besides that, the user must specify an instance diagram, which will guide in the generation of the executable simulation model.

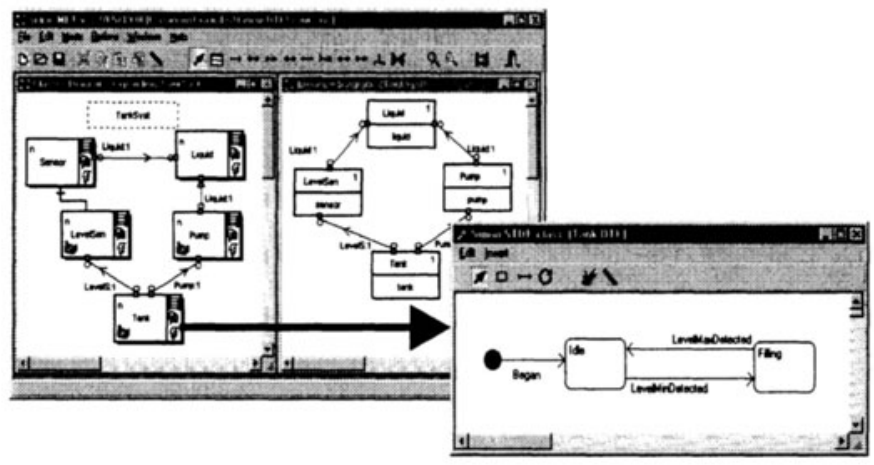

Fig. 2: Example of SIMOO-RT model.

SIMOO-RT extends original SIMOO [18], incorporating on it specific features that allow definition of temporal requirements, like deadlines and periodic operations. This environment encourages the use of state machines to describe the model behavior. SIMOO-RT also offers support to make automatic code generation for a real-time operating system, where the adopted target language is $\mathrm{AO} / \mathrm{C}++[19]$. 


\subsection{SysObj}

SysObj is a CASHE (Computer Aided Software Hardware Engineering) tool that implements the aspects of the methodology related with the automatic object generation and QM computation. It accepts, as input, a file containing the DFD description of system requirements, as provided by the Paradigm Plus CASE tool, or modified in order to include testability features. Additionally, task and task association weights can be introduced, to reflect either complexity, or timing requirements. It provides, as output, a discrete set of architectural solutions, one per number of system objects, as well as the values of the different QMs that guide the design team in the selection of the final architecture. At present, SysObj is embedded in SIMOO-RT, in the MOSYS environment [7].

\section{Case-study}

The system, in which the case study is embedded, is an industrial production line. The case study is based on the design of the real-time control system, responsible for the automation of the production line [20]. Here we will only address the aspects that are relevant for methodology assessment. The actual architectural solution for the centralized control system (referred as "archit.1") has been derived using an $\mathrm{OO}$ Modeling technique (fig. 3). In the complete development, system architecture has been derived using design-oriented metrics and criteria for deciding the best allocation of processes and attributes within objects.

The analysis to be carried out aims at demonstrating that gains can be obtained when optimization algorithms are used for functionality distribution. For doing so, the system functionality and system context are viewed as one global system. In the actual implementation (archit.1, Fig. 3), the control system makes use of a set of sensors distributed along the production line. Control is carried out based on the values provided by the sensors.

The sensors used in the actual implementation are passive sensors. The objective of the study is to compare this implementation with alternative implementations assuming a distributed processing, based on the use of smart sensors. For that, intelligence must migrate from the central processor to the different sensors.

Two strategies are used for assigning intelligence to the sensors. The traditional approach would be to migrate intelligence from the class that represents the sensor processor to the class that represents the sensor. We refer this as "archit.3". In the proposed approach, the original E-DFD is modified according to the new objective, i.e., to distribute intelligence within the actors. After that, the remaining functionality distribution is carried out by SysObj, using optimization algorithms, QM computation and decision criteria. A novel architectural solution ("archit.2") is then automatically generated.

The automatic generation of system architecture, as carried out with SysObj, is shown in Fig. 3 for archit.1. In this approach, we have retained one of the customer constraints, that is, one processing unit for each machine, in the production line. Four main objects, responsible for all data processing and line control constitute the generated architecture. Sensors have been defined as actors. 


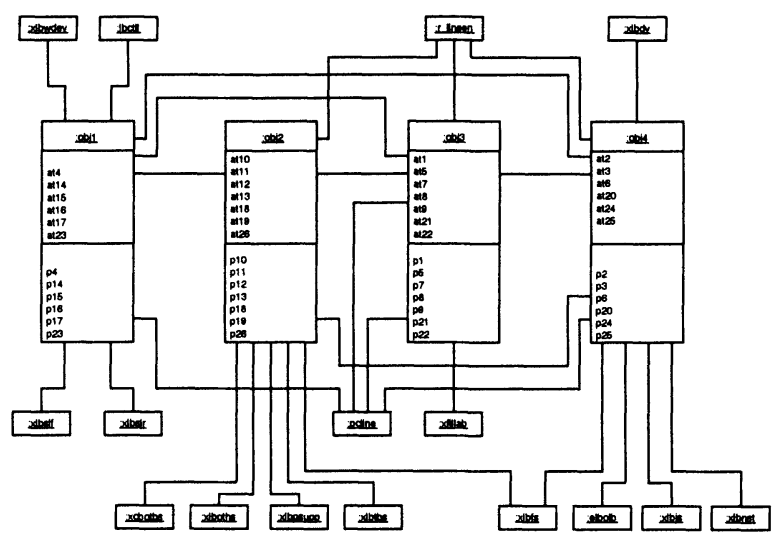

Fig.3 - Automatically generated OO diagram (archit.1)

As referred, in the actual (centralized) implementation, there is no 'intelligence' associated with sensors. In archit.2, tasks within objects are automatically reassigned, so objects of similar complexity result. Again, the selected architectural solution corresponds to a 4-object architecture. As expected, when comparing 4object archit.1 and 2, QM computation by SysObj shows that the system associated with the distributed architecture exhibits higher system coupling (from 58.7 to $63.0 \%$ ) and lower average object autonomy (from 1.04 to 0.83 ). Formal QM definition has been presented elsewhere [6]. However, these slight modifications in metrics values do not have a significant impact on architecture quality. In archit.3, tasks associated with sensor's data processing have been manually moved to the corresponding objects. No other modification on the four main objects of archit.1 has been made. Now, it is to be expected that an unbalanced architecture may become visible by $\mathrm{QM}$ evaluation.

Three QMs quantifying individual object characteristics are used for comparing the three resulting architectures, namely, Cohesion, Autonomy and Object Relative Weight (ORW). The first two metrics describe the structural aspects of the architecture (namely, the strengths of object associations), while the third metric values object comparisons, in terms of their weights, which quantify either complexity, or timing response. The results of the evaluation are represented in tables 1-3. In fig. 4, methods (or task) redistribution, in archit.2 and 3, is depicted. 
Table 1 -Central processing architecture (archi.1)

\begin{tabular}{|l|r|r|r|}
\hline Archit. 1 & \multicolumn{3}{|c|}{ Q. metrics } \\
\hline & cohesion (\%) & autonomy & ORW (\%) \\
\hline Object 1 & 2.82 & 1.1 & 23.08 \\
\hline Object2 & 4.14 & 1.08 & 26.92 \\
\hline Object3 & 3.85 & 1.25 & 26.92 \\
\hline Object 4 & 2.4 & 0.71 & 23.08 \\
\hline Average & 3.3 & 1.04 & \\
\hline Std. Dev. & 0.71 & 0.2 & 1.92 \\
\hline
\end{tabular}

Table 2 - Automatically generated architecture for distributed processing (archit.2)

\begin{tabular}{|l|r|r|r|}
\hline Archit.2 & \multicolumn{3}{|c|}{ Q. metrics } \\
\hline & cohesion (\%) & autonomy & ORW (\%) \\
\hline Object 1 & 4.76 & 1.4 & 28.57 \\
\hline Object2 & 2.24 & 0.5 & 23.81 \\
\hline Object3 & 2.86 & 0.55 & 23.81 \\
\hline Object 4 & 3.06 & 0.9 & 23.81 \\
\hline Average & 3.23 & 0.84 & \\
\hline Std. Dev. & 0.93 & 0.36 & 2.06 \\
\hline
\end{tabular}

Table 3 - Manually modified architecture for distributed processing (archit.3)

\begin{tabular}{|l|r|r|r|}
\hline Archit. 3 & \multicolumn{3}{|c|}{ Q. metrics } \\
\hline & cohesion (\%) & autonomy & ORW (\%) \\
\hline Object 1 & 3.49 & 1.1 & 28.57 \\
\hline Object2 & 1.43 & 0.25 & 14.29 \\
\hline Object3 & 4.76 & 1.25 & 33.33 \\
\hline Object 4 & 3.3 & 0.82 & 23.81 \\
\hline Average & 3.24 & 0.85 & \\
\hline Std. Dev. & 1.19 & 0.38 & 7.04 \\
\hline
\end{tabular}

As it can be seen through ORW values, archit.1 (benchmark) and 2 exhibit balanced objects, as automatic graph partitioning has been carried out favoring (1) object balance and (2) graph min-cut. In contrast, archit.3 (the usual solution) creates especially one object (obj.2) clearly unbalanced, with low cohesion and autonomy (i.e., strongly dependent on the remaining objects) and lower ORW. This is reflected on the higher standard deviation values. Conversely, obj.3 is more cohesive and autonomous than the average $(25 \%)$.

As shown in Fig. 4, 5 tasks have been removed from the original system (archit.1), which is the centralized architecture. However, the optimized distributed architecture (archit.2) corresponds to a class (or object) definition for which methods (or task) assignment to the objets greatly differs from the one in archit.1. In fact, e.g., object 1 in archit. 2 has assigned to it methods originally assigned to all the 4 objects of archit.1. This example highlights the fact that manually moving functionality to the external actors can produce a poor architectural solution. As shown in Fig. 4, obj. 2 in archit. 3 has now only 3 remaining methods assigned to it, as compared to the original 7, which justifies the quantitative values depicted in table 3 . 


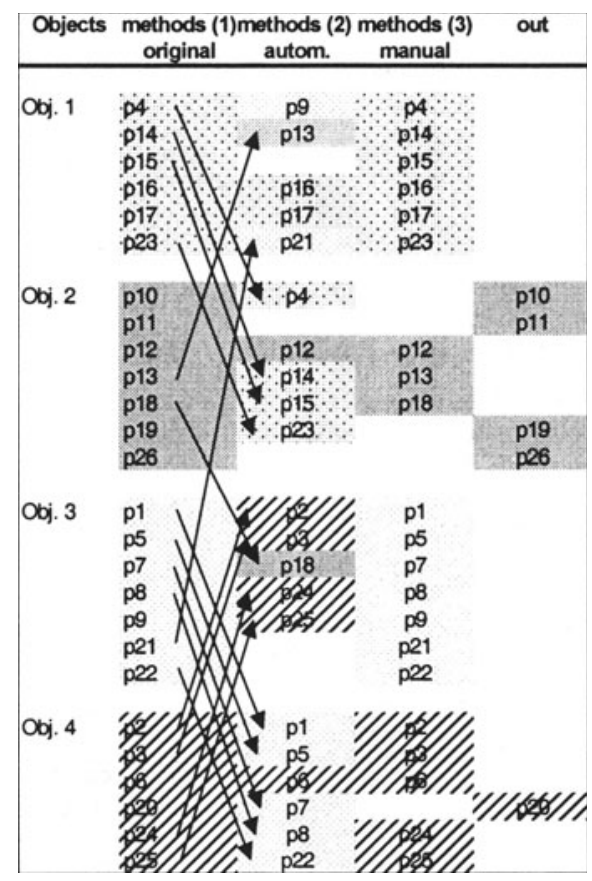

Fig.4 - Methods reassignment. In archit.2 and 3, p10, 11, 19, 20 and 26 are moved out of the system.

\section{Conclusions}

In conclusion, this paper shows that functionality distribution among embedded objects of a system architecture is a critical issue for the quality of the resulting architecture. Significant gains can be obtained when functionality distribution uses optimization criteria, such as object balance (in terms of task complexity, or execution time) and autonomy. Centralized versus distributed architectures have been compared, allowing tradeoff analysis to be performed. In order to generate a distributed architecture, starting from a centralized one, two alternatives have been used. First, functionality is removed from the processing class, to external actors, manually. Second, the entire architecture is re-generated to comply with distributed processing. Automatic architecture generation uses OOriented Paradigm and UML, in conjunction with an extension of data flow diagram (E-DFD) conveying timing information have been used for system requirement formalization. Two proprietary tools have been used to implement the methodology. SIMOO-RT is used as graphical interface for direct Use-Case and E-DFD diagram constructions and for simulation. SysObj is used for architecture generation and quality assessment. Resident Quality Metrics and Criteria are used. Finally, it was shown that task assignment in the object definition of the optimized distributed architecture greatly 
differs from the one in the manually modified architecture. This conslusion clearly justifies the usefulness of using a methodology for automatic generation of system architectures, valuing characteristics such as object balance and enhanced autonomy, which in turn favors performance, parallelization and testability.

\section{References}

[1] L.Lavagno, A. Sangiovanni-Vincentelli, H. Hsieh, "Embedded System Codesign", Nato ASI-Series, Kluwer Academic Publishers, 1996.

[2] R.K. Gupta, Y. Zorian, "Introducing Core-Based System Design", IEEE Design \& Test of Computers, vol. 12, pp. 15-25, Sept./Oct. 1997.

[3] M. Keating, P. Bricaud, "Reuse Methodology Manual for Systems-on-a-Chip Designs", $2^{\text {nd }}$. Ed., Kluwer Acad. Pub., 1999.

[4] H.P.E. Vranken, M.F. Wittman, R.C. van Wuijtswinkel, "Design for Testability in Hardware-Software Systems”, IEEE Design \& Test, vol. 13, No. 3, pp. 79-87, 1996.

[5] O.P. Dias, J. Semião, I.M. Teixeira, J.P. Teixeira, C.E. Pereira, "Introducing Test Requirements in Real Time Systems at Architectural Level", Proc. $14^{\text {th }}$. Conf. On Design of Integrated Systems (DCIS), pp. 651-656, Nov., 1999.

[6] O.P. Dias, I.C. Teixeira, and J.P. Teixeira, "Metrics and Criteria for Quality Assessment of Testable Hw/Sw System Architectures", Journal of Electronic Testing, Theory and Application (JETTA), vol. 11, n. 1/2, pp. 149-158, Kluwer Pub., February/April, 1999.

[7] O.P. Dias, L.B. Becker, I.M. Teixeira, J.P. Teixeira and C.E. Pereira, "MOSYS: A Methodology for Automatic Object Identification from System Specification", Proc. 3rd. IEEE Int. Symp. on Object-Oriented Real Time Distributed Comp. (ISORC), March, 2000.

[8] O.P. Dias, I.C. Teixeira, J.P. Teixeira and C.E. Pereira, "Performance and Test-Oriented Metrics and Criteria for Hw/Sw Design at System Level", Proc. of the Design and Diagnostics of Electronics and Systems Workshop (DIPES), pp. 219-225, Polland, 1998.

[9] L. Becker, C.E. Pereira, O.P. Dias, I.M. Teixeira, J.P. Teixeira, "On Identifying and Evaluating Object Architectures for real-Time Applicstiond", Proc. 6th. IFAC Workshop on Algorithms and Architectures for Real-Time Control (AARTC), Spain, May, 2000.

[10] J. Rumbaugh, et al., "Object-Oriented Modeling and Design", Prentice Hall, 1991.

[11] G. Booch, "OO Design with Applications", The Benjamin/Cummings Pub. Co., Inc., 1991.

[12] J. Rumbaugh, "OMT papers", 1994/95, http://www.rational.com/support/techpapers/omt.

[13] M. Keating, "Measuring Design Quality by Measuring Design Complexity", Proc. IEEE 1st. Int. Symposium on Quality of Electronic Design (ISQED), pp. 103-108., March, 2000.

[14] "Software Quality Metrics for Object-Oriented System Environments", 24 Sw Assurance Technology Center (SATC), 1997, http://www.satc.gsfc.24.gov/SACT/PAPERS

[15] "Complexity Measurements", http://www.mccabe.com/appnote/complex.html.

[16] "UML - Unified Modeling Language", version 1.0, Rational Software Corporation, January, 1997, http://www.rational.com.

[17] L.B. Becker and C.E. Pereira, From Design to Implementation: Tool Support for the Development of Object-Oriented Distributed Real-Time Systems. In Proc. of 12th Euromicro Conference on Real-Time Systems. Stokholm, Sweeden, June 2000. pp. 108115.

[18] B. Copstein, F. Wagner, and C. Pereira, SIMOO - An Environment for the Object-Oriented Discrete Simulation. In: ESS'97 - 9th European Simulation Symposium. Passau, Germany, October 1997. Proceedings, Society for Computer Simulation, 1997.

[19] C. Pereira, "Real Time Active Objects in C++/Real-Time UNIX", Proc. of ACM SIGPLAN Workshop on Languages, Compiler, and Tool Support for Real-Time Systems. 1994. 
[20] R. Ribeiro, O.P. Dias, J.P. Teixeira, I.M.Teixeira, "Automation and Real-Time Control of a Wine Bottling Production Line: an Object Oriented Based Hw/Sw Co-Design Case Study", Proc. $8^{\text {th }}$. IFAC Conf. on Large Scale Systems (LSS), vol. 2, pp. 1211-1216, 1998. 